\title{
DISEÑANDO UN NUEVO MODELO ECONÓMICO: PROPUESTAS DESDE EL DERECHO CONSTITUCIONAL Y LA ECONOMÍA FEMINISTA SOBRE EL CUIDADO Y LA IGUALDAD FRENTE A LA CRISIS COVID-19'
}

\section{Designing a new economic model: proposals from constitutional law and feminist economics on care and equality in the face of the COVID-19 crisis}

\author{
ANA MARRADES PUIG \\ Universitat de València \\ Ana.I.Marrades@uv.es
}

Cómo citar/Citation

Marrades Puig, A. (2020).

Diseñando un nuevo modelo económico: propuestas desde el derecho constitucional y la economía feminista sobre el cuidado y la igualdad frente a la crisis COVID-19.

IgualdadES, 3, 379-402

doi: https://doi.org/10.18042/cepc/lgdES.3.04

(Recepción: 01/07/2020; aceptación tras revisión: 06/10/2020; publicación: 18/12/2020)

\section{Resumen}

La crisis provocada por la pandemia COVID-19 ha puesto de manifiesto las debilidades del sistema económico, sanitario y de cuidados. Ha supuesto también un punto de inflexión para replantearnos un cambio cultural — que ya se estaba

\footnotetext{
El presente trabajo se ha realizado en el marco del proyecto «Generando un interpretación del derecho en clave de igualdad de género", del Programa Estatal de $\mathrm{I}+\mathrm{D}+\mathrm{i}$ Orientada a los Retos de la Sociedad RTI2018-100669-B-I00. Así como también como resultado de las investigaciones propuestas por la Cátedra de Economía Feminista de la Universitat de València para el curso 2020.
} 
fraguando y que ahora deviene más necesario que nunca, acompañado de cambios en el modelo económico y de nuevas formas de diseñar las políticas públicas - que reorganice el sistema de valores que rigen en nuestra sociedad, resituando el valor del cuidado y la atención a las personas por encima de otros intereses económicos. Este trabajo pretende, por un lado, reflexionar sobre la necesidad de entender la economía desde otros enfoques, teniendo como fundamento la universalización de los derechos fundamentales y la acción del derecho inspirada en la ética del cuidado, de la igualdad y de la solidaridad, y por otro, ofrecer propuestas desde la economía feminista y el derecho constitucional para afrontar la nueva era post COVID-19.

\section{Palabras clave}

Crisis COVID; cuidados; economía feminista; derechos fundamentales.

\section{Abstract}

The crisis caused by the COVID-19 pandemic has highlighted weaknesses in the economic, health and care system. It has also been a turning point to rethink a cultural change — which was already being forged and which is now becoming more necessary than ever, accompanied by changes in the economic model and new ways of designing public policies - that reorganizes the system of values that govern our society, restoring the value of care and attention to people above other economic interests. This work aims, on the one hand, to reflect on the need to understand the economy from other approaches, with the basis of the universalization of fundamental rights and the action of law inspired by the ethics of care, equality and solidarity, and on the other hand, to offer proposals from the feminist economy and the constitutional law to face the new post-COVID-19 era.

\section{Keywords}

COVID crisis; care; feminist economy; fundamental rights. 


\section{SUMARIO}

I. LA CRISIS DEL COVID-19 Y LA VULNERABILIDAD HUMANA. II. EL MODELO ECONÓMICO CONSTITUCIONAL: ¿QUÉ TENEMOS Y A QUÉ ASPIRAMOS? III. LOS CUIDADOS Y LAS CUIDADORAS: 1. La ética del cuidado. 2. El colectivo de las cuidadoras. IV. LAS PROPUESTAS DESDE LA ECONOMÍA Y EL DERECHO CONSTITUCIONAL: 1. Desde la economía feminista: 1.1. Una renta de cuidados. 1.2. Un sistema estatal de cuidados. 2. Desde el derecho constitucional: 2.1. Los derechos sociales fundamentales. 2.2. La jurisprudencia en igualdad. V. A MODO DE EPÍLOGO: ¿̇UNA NUEVA ERA? VI. BIBLIOGRAFía.

\section{LA CRISIS DEL COVID-19 Y LA VULNERABILIDAD HUMANA}

En los primeros periodos de la crisis COVID-19 coincidentes con las primeras semanas de vigencia del estado de alarma ${ }^{2}$, el foco de interés se situaba en la tensión entre la seguridad/salud y la libertad. Entrando ya en la fase de desescalada el foco fue desplazándose hacia otra tensión, la que iba emergiendo entre la seguridad para la salud de las personas junto con el buen funcionamiento de los servicios sanitarios y la economía, y esta tensión vino para quedarse, ya que el daño a la economía se augura irreparable.

¿Es posible conciliar ambos extremos, es decir, la protección de la salud de toda la población y el crecimiento económico? O incluso más allá de la vuelta a la normalidad de la situación sanitaria y la superación de las medidas de protección después de la desescalada total, ¿̇es posible recuperar la estabilidad económica y hacerlo sin dañar las conquistas alcanzadas en derechos sociales? Es la pregunta o preguntas cruciales para que los países, azotados por la pandemia en mayor o menor medida, puedan seguir adelante, en especial los países que se reconocen como Estados sociales y democráticos de derecho.

Diseñar consiste en hacer un plan detallado para la ejecución de una acción o una idea. La idea o el objeto de este trabajo es apuntar algunas reflexiones sobre qué modelo económico debe observarse o qué posibles modificaciones

2 El Gobierno aprobó el 14 de marzo de 2020 declarar el estado de alarma (Real Decreto 463/2020) en todo el territorio español para afrontar la situación de emergencia sanitaria provocada por la COVID-19 (disponible en: https://bit.ly/3paln2h). 
deben introducirse para que podamos afrontar la crisis económica y de derechos que está emergiendo y se proyecta como una amenaza también para el futuro. Ahora más que nunca la economía y el derecho deben entenderse.

Para ello debemos partir de algunas obviedades que la crisis COVID-19 ha puesto de manifiesto.

La extrema vulnerabilidad del ser humano es la primera de ellas, junto con el valor de la vida y todo lo que ayuda a sostenerla: el cuidado a las personas debe revalorizarse en el marco de un Estado social de derecho. Somos vulnerables e interdependientes, y en circunstancias de fragilidad necesitamos de los demás.

Por tanto, y como consecuencia de lo anterior, en segundo lugar, el colectivo de las personas cuidadoras debe estar altamente reconocido desde una perspectiva económica, pero también social y política. Como dice Alain Touraine, «estamos entrando en un nuevo tipo de sociedad, la sociedad de los servicios, pero de los servicios entre humanos», por lo que esta crisis revalorizará la categoría de los cuidadores, cuya labor debe estar bien remunerada (Bassets, 2020).

Tercero, el colectivo de las personas cuidadoras está altamente generizado. Un $70 \%$ del sector sociosanitario está formado por mujeres. Y no solo desde el ámbito profesionalizado, sino también en el ámbito privado: las cuidadoras de la familia, de las personas menores, mayores, enfermas y dependientes también son mujeres ${ }^{3}$. Por eso su productividad (en términos mercantiles ${ }^{4}$ ) ha disminuido y eso ha provocado un incremento de la desigualdad existente, situando a las mujeres que se han ocupado del cuidado durante este periodo en una posición más complicada en el ámbito laboral.

Por último, el cuidado sea o no remunerado, debe ser revalorizado en la sociedad y contar para la economía (Moltó y Uriel, 2008: 228) porque del mismo depende la supervivencia de la sociedad en condiciones dignas, y para ello se requiere un cambio cultural que revalorice lo verdaderamente importante: la salud de las personas en un entorno sostenible, sin contaminación, con unas condiciones vitales garantizadas. También es obvio que para esto hay que invertir en los cuidados, hay que invertir en sanidad y hay que educar en la transmisión de los valores de la solidaridad, la diversidad, la igualdad y la corresponsabilidad.

3 «Comunicado» de la Cátedra de Economía Feminista ante la COVID-19 (disponible en: https://bit.ly/3p5aDCx).

4 La cantidad de trabajo no disminuye, pero sí la productividad en términos de mercado, ya que lo que se da es un trasvase de horas de trabajo entre la esfera mercantil y la de cuidados no remunerados. 
Educación, nuevos valores, cambios de paradigmas culturales y económicos, son propósitos que están siendo reiterados en esta época en crisis. Touraine plantea como una de las decisiones fundamentales para Europa «la liberación por medio de las mujeres», es decir, «el derrumbamiento de la razón como aspecto central y la recomposición de los afectos en torno a la razón y la comunicación, una sociedad del care (de los cuidados)». En definitiva, plantear un nuevo modelo de cultura y de sociedad que priorice los cuidados. Tal vez construir una épica del cuidado que diría Irene Vallejo (2020), que señale quiénes son ahora las verdaderas heroínas y héroes.

Según propone Katrine Marçal (2016:127), si se quiere preservar el cuidado a las personas en nuestra sociedad, este debe ocupar un papel central y debe ser apoyado con los recursos económicos necesarios. De este modo se recuperarán otros bienes esenciales para la economía, no solo los productivos deben contar, sino también los reproductivos. Solo así se pueden reconciliar los distintos valores democráticos.

\section{EL MODELO ECONÓMICO CONSTITUCIONAL: ¿̇QUÉ TENEMOS Y A QUÉ ASPIRAMOS?}

En el marco de su preámbulo, la Constitución española comienza su redacción apelando a la voluntad de la Nación española de "garantizar la convivencia democrática dentro de la Constitución y de las leyes conforme a un orden económico y social justo». El modelo económico - o Constitución económica - queda definido en el título VII, «Economía y Hacienda», aunque no solo, porque hay otros aspectos que lo definen en el título I, como los derechos sociales y los principios rectores de la política social y económica.

Entonces, ¿de qué modelo económico parte la Constitución española? Parece ser que aunque conjuga elementos sociales y concede un margen de intervención estatal, la Constitución de 1978 establece un patrón de economía de mercado próximo al modo neoliberal de entender la economía. Como ya explicaba López Guerra (1983: 17) «una de las peculiaridades de la Constitución española, frente a la gran mayoría de los textos constitucionales de otros países es que - pese a los comentarios que a veces se han hecho- se pronuncia explícitamente en favor de un modelo económico determinado, que es, en definitiva, el de la economía de mercado; modelo que, con todas las precisiones que puedan hacerse, se corresponde, en nuestro lenguaje, con el sistema "capitalista"». Si bien la acción de algunos Ejecutivos a través de ciertas políticas públicas, el desarrollo legislativo y determinada interpretación jurisprudencial han ido permitiendo nuevos enfoques con tintes más sociales. Es obvio que ello ha dependido y dependerá en mayor o menor medida de la 
orientación política del Gobierno en cada legislatura. Sin embargo, en todo caso sigue predominando el diseño trazado por la economía convencional.

La economía feminista se aparta de la forma convencional de entender la economía para proponer otro modelo que aspire a lograr un orden económico justo, que asegure una digna calidad de vida para todas las personas. Lourdes Benería et al. sintetizan dos posibles enfoques de este modelo, el de los derechos y el de las capacidades, en su obra magistral (2018).

El enfoque de las capacidades es uno de los discursos más potentes contra el planteamiento neoliberal. Este enfoque económico postulado por Amartya Sen y Martha Nussbaum se fundamenta sobre políticas dirigidas a suprimir los obstáculos que encuentran las personas en su vida para poder vivirla en libertad de la mejor manera posible. «Las capacidades representan el potencial multidimensional de cada individuo. Una capacidad es la posibilidad de ser y de hacer lo que la persona quiere ser y hacer» (Benería et al., 2018: 65). Este concepto se identifica de manera bastante plausible con el libre desarrollo de la personalidad que la Constitución española reconoce en el art. 10 como uno de los fundamentos del orden político y de la paz social.

Martha Nussbaum desarrolló el enfoque de las capacidades ajustándolo a una teoría de la justicia y de la potenciación universal de las capacidades de las mujeres. Elaboró una lista irreducible de capacidades, entre las que necesariamente está la de vivir una vida digna con salud y con libertad de acción, libre de violencia de género. Este, que sería el resumen esencial de esa lista de diez capacidades, tiene como premisa, explican las autoras (ibid.: 66), la idea de que «ser humano o humana implica ser un ente físico con cuerpo, ser cuidado o cuidada y poder cuidar a otras personas». Nussbaum $(2003,2004)$ está poniendo el foco de nuevo, en el cuidado, y por otro lado está exigiendo que estas capacidades sean universales, es decir, que estén garantizadas constitucionalmente en todas partes. El reclamo de la inclusión de las capacidades en el texto constitucional resulta evidente y consecuentemente se nos plantea la siguiente pregunta: jes posible conciliar la garantía de estas capacidades con el modelo económico de nuestra Constitución? La respuesta puede ser afirmativa si se conjuga el art. 10 — la dignidad humana como eje y el libre desarrollo de la personalidad como posibilidad- con las garantías de los derechos sociales y otros posibles ingredientes, que — como más adelante se verá- tienen que ver con la revisión de algunos derechos sociales o principios rectores como derechos fundamentales o el reconocimiento de algún otro derecho fundamental.

Esta última reflexión enlaza con el otro enfoque económico: el enfoque de los derechos humanos. Este enfoque desarrollado por Balakrishnan y Elson (2011) parece que podría tener mayor incidencia en la modificación de las políticas neoliberales, ya que permite realizar su evaluación a partir de los derechos, considerando que existen unos mínimos esenciales en los derechos 
económicos y sociales que es preciso preservar en todos los procesos económicos (Benería et al., 2018: 72).

Resulta evidente que ambos enfoques se complementan y tienen importantes objetivos comunes, como por ejemplo la satisfacción de las necesidades humanas para una vida digna. Así podríamos considerar que nuestra Constitución se puede interpretar en clave feminista y de igualdad real también en relación con el modelo económico desde el art. 10, que sitúa la dignidad como eje de los derechos y el desarrollo libre de la personalidad como fundamento del orden político, junto con el artículo 9.2, que promueve lo necesario para que la igualdad sea efectiva. A ello habría que añadir una revisión de los derechos del capítulo III y algunos del capítulo II en tres sentidos: ampliación de la relación de derechos fundamentales, de manera que algunos derechos no considerados como fundamentales por no estar ubicados en la sección primera del capítulo II lo sean; utilización efectiva de la cláusula hermenéutica del artículo 10.2 para ampliar el contenido de algunos derechos, e inclusión de algún nuevo derecho.

El objetivo de revisar y reinterpretar la Constitución en clave de género e igualitaria obedece principalmente a la obligación de hacer frente a las situaciones de necesidad económica que habrá que solucionar, así como a las graves discriminaciones entre personas afectadas por la epidemia en España.

Como argumenta Ramonet (2020), incluso grandes defensores del modelo neoliberal están tomando conciencia de los tremendos errores del neoliberalismo al considerar que hay que construir una sociedad justa «mediante un nuevo modelo definitivamente más justo, más ecológico, más feminista, más democrático, más social, menos desigual». Sin embargo, «es poco probable que una vez vencido el azote, mantengan sus propósitos. Sería una auténtica revolución [...]. Y un virus, por perturbador que sea, no sustituye a una revolución [...]. No podemos pecar de inocentes. Las luchas sociales seguirán siendo indispensables [...], los poderes dominantes, por mucho que se hayan tambaleado, se esforzarán por retomar el control [...] con mayor violencia, si cabe. Tratarán de hacernos regresar a la vieja "normalidad". O sea, al Estado de las desigualdades permanentes», desigualdades que ahora serán más profundas y evidentes. Las desigualdades económicas por supuesto, pero también las desigualdades que se basan en el género y en la forma en que se estructura la sociedad, y las discriminaciones por razón de sexo. Todas están relacionadas por ese fenómeno del que tantas veces se ha alertado de la feminización de la pobreza, y no solo en países en vías de desarrollo, sino también en el nuestro y en el marco de los países de la Unión Europea.

Por otro lado, la desigualdad hay que analizarla en relación con otros dos conceptos: la conciliación y la corresponsabilidad. La crisis de la COVID-19 ha puesto en evidencia más que nunca que la carga de los cuidados y la aten- 
ción a las personas en la familia la siguen llevando las mujeres. Parece que de nuevo la conciliación sea cosa de ellas y que la corresponsabilidad se haya ido diluyendo. Con el cierre de los centros educativos y las niñas y los niños en los hogares, las mujeres manifiestan la imposibilidad de conciliar. Su productividad, junto con su salud física y mental, se están viendo ya afectadas y ello tendrá consecuencias profesionales, sin lugar a dudas.

Todo apunta a una quiebra del principio de igualdad, lo que exige un modelo económico diferente al neoliberal. A ello cabe añadir que los modelos de producción del que se nutre el sistema económico que conocemos han estado saqueando la naturaleza y modificando el clima, alterando la biodiversidad y creando las condiciones objetivas para que nuevos virus y nuevas enfermedades aparezcan. Este es pues otro motivo para intentar sustituirlo. Alicia Puleo describe el neoliberalismo global como «un avatar del patriarcado originario", desde el ecofeminismo que denuncia la falsa e interesada definición de felicidad como acumulación de bienes materiales que van lastrando la existencia de las personas y del medio que habitamos (2019: 13). Este movimiento analiza el dominio de la sociedad patriarcal sobre la naturaleza y las mujeres a través de sus diversas manifestaciones históricas. Ya desde la filosofía clásica se asimilaba mujer y naturaleza, un ser para otros, asociando a los hombres con la cultura. Esta idea nos ha estado acompañando a través de los siglos y la seguimos viendo reflejada hoy en la asunción pretendidamente espontánea de las tareas del cuidado por las mujeres.

\section{LOS CUIDADOS Y LAS CUIDADORAS}

\section{LA ÉTICA DEL CUIDADO}

El concepto de ética del cuidado surge en la década de los ochenta a partir de los estudios de Carol Gilligan (1985: 13) sobre el desarrollo de la psicología evolutiva en mujeres y hombres y los dos diversos modos de hablar de problemas morales, de las dos diversas éticas, la de la justicia y los derechos; $\mathrm{y}$, la del cuidado y la responsabilidad.

Gilligan (1985: 266) explica que, aunque ambas éticas se basan en la igualdad, la moral de los derechos se centra en la comprensión de la imparcialidad, mientras que la ética de la responsabilidad se centra en el reconocimiento de las diferencias de necesidad. Ambas se complementan (ibid.: 280) sin que la ética del cuidado deba suponer «dependencia o subordinación», y ambas constituyen "dos perspectivas morales que organizan tanto el pensamiento como los sentimientos y empoderan al sujeto a tomar diferentes tipos de acciones tanto en la vida pública como privada» (1993:209). 
Aunque ya en otras ocasiones me he pronunciado ampliamente (Marrades Puig, 2016, 2018, 2019) sobre este concepto y sus aspectos más relevantes en los estudios feministas, merece la pena resituarlo actualmente tras dos décadas del inicio del siglo XXI y al comienzo de la que probablemente podría ser una nueva era.

Con el soporte de la teoría de Victoria Camps (2000: 69-77), que marca una guía de lo que supone entender el cuidado al inicio del siglo xxI, y con los cambios acontecidos en estas dos primeras décadas, podemos configurar el cuidado como un elemento indispensable para una sociedad más justa que debe complementarse con otro elemento; la corresponsabilidad. Así lo han entendido los poderes públicos, y en concreto, a modo de ejemplo, el segundo Gobierno de la XII legislatura aprobó el «Real Decreto Ley 6/20195, de 1 de marzo, de medidas urgentes para garantía de la igualdad de trato y de oportunidades entre mujeres y hombres en el empleo y la ocupación", con el fin de ir ajustando la duración igual de los permisos de maternidad y paternidad.

Con la crisis producida por la COVID-19 han saltado las alarmas acerca de la necesidad de revalorizar el cuidado, poner la atención a las personas en el centro y llamar a la solidaridad y corresponsabilidad. Sin embargo, de nuevo, algunas voces feministas alertan sobre la precaución que hay que observar al acentuar la importancia del cuidado por el riesgo de seguir recayendo en las mujeres. Incluso ante la coincidencia demostrable de que los países gobernados por mujeres han gestionado mejor la crisis sanitaria (Kohan, 2020) hay un empeño en matizar que la causa de la posible mejor gestión no debe descansar en rasgos diferenciales y que puede deberse a muchas otras variables. Cierto, pero también lo es que, debido a una educación determinada, estamos mejor dotadas de cualidades para la empatía, la comunicación y la gestión de conflictos de cuidados. Una larga trayectoria histórica nos avala.

Es preciso insistir en que no hablamos de cualidades innatas sino aprendidas, por lo que de la misma manera que las hemos aprendido las mujeres pueden aprenderlas los hombres. Consecuentemente pueden extenderse a toda la sociedad o todas las sociedades a través de la educación y de la reconstrucción del sistema social que hace muchos años se encargó de atribuir roles y cualidades a las personas en función de su sexo. Si nacías mujer estabas asignada a los cuidados y si nacías varón estabas exento de su aprendizaje y de su práctica. Así, aunque se ejerciera profesionalmente, por ejemplo, un profesional de la medicina

Disponible en: https:/www.boe.es/boe/dias/2019/03/07/pdfs/BOE-A-2019-3244.pdf.

6 Me refiero al proceso de socialización que a lo largo de la historia y en el seno de una estructura social patriarcal ha preparado a las mujeres para las tareas del cuidado. Existe una amplia bibliografía sobre ello que a modo de ejemplo puede citarse, como los trabajos de Carol Gilligan y Victoria Camps. 
curaba, decidía sobre una intervención o tratamiento, pero no cuidaba; eso lo hacía la enfermera. Con el tiempo se fue equilibrando el ámbito profesional, pero no del todo: según el informe de ONU Mujeres ante la crisis de la COVID (Bathia, 2020), la presencia de mujeres en los foros internacionales de estudio y gestión de medidas ante la crisis es significativamente menor a la de los hombres; por tanto, el mando de la gestión sigue siendo masculino. Por otro lado, en los hogares las mujeres siguen asumiendo la carga de los cuidados. La crisis sanitaria COVID-19 ha intensificado la brecha de cuidados y la balanza se ha decantado claramente por aumentar la carga en las mujeres.

\section{EL COLECTIVO DE CUIDADORAS}

Al referirme al colectivo de cuidadoras estoy pensando en dos grupos principalmente: las mujeres de la familia que cuidan de las personas que tienen a su cargo (menores, mayores, enfermas, dependientes), y las cuidadoras externas asalariadas.

La crisis ha salpicado de manera desigual a ambos grupos de mujeres, y siempre en sentido negativo. Las mujeres han tenido que asumir el peso principal de los cuidados en los hogares y además sin la ayuda externa, en caso de que existiera, ya que el temor al contagio y la debida precaución suspendió provisionalmente los contratos. Por otro lado, y por la misma razón, las cuidadoras profesionales vieron suspendidos sus trabajos y reducidos sus ingresos.

El peso principal de los cuidados ha repercutido muy negativamente en la conciliación, a pesar de la falacia del teletrabajo que parecía propiciarlo, y la corresponsabilidad ha brillado por su ausencia.

Un estudio muy reciente de la Universitat de Valencia (Benlloch y Aguado, 2020) sobre la conciliación en el confinamiento por el estado de alarma decretado ante la pandemia COVID-19 ha detectado importantes problemas. Entre ellos, y principalmente, que las mujeres que teletrabajan están cuidando al mismo tiempo, asumiendo ellas las tareas del cuidado y, a la vez, «facilitando que sus parejas trabajen o teletrabajen». El hecho de tener «el trabajo en casa» no aligera la carga, sino al contrario; la supuesta flexibilidad se convierte en «una demostración continua y un ejercicio de responsabilidad para con sus superiores». Por otro lado, las madres se han convertido prácticamente también en maestras, realizando ellas mayoritariamente el seguimiento escolar de los hijos e hijas en edad educativa. Sin embargo, y como factor positivo, en algunas parejas se ha observado una mayor disposición por parte de los hombres a realizar tareas que no solían realizar antes (poner lavadoras, cocinar, ir a la compra, compartir horas de juego con los niños...). Ahora bien, la gestión doméstica principalmente la siguen llevando las mujeres. La carga de doble y triple jornada es tal que deben optar por trasnochar o trabajar 
durante la madrugada para provechar las horas de silencio y de concentración en los trabajos que lo requieran. Por otro lado, hay que añadir que la productividad de trabajos académicos de mujeres también se ha reducido.

Es imprescindible recuperar la política para las personas y con enfoque de género, es imprescindible recuperar el sentido de la corresponsabilidad porque si no se toma en serio, las políticas de igualdad y las conquistas a través de ellas alcanzadas pueden retroceder significativamente.

María Ángeles Durán (2018: 481) apunta que las predicciones de demanda de cuidados tienen un significado especial por la urgencia de encontrar alternativas a los problemas que no podrán resolver ni el mercado ni los hogares ni el Estado. Si se prevé que para 2050 la demanda aumente un $50 \%$ más que en la actualidad, resulta indispensable tomar en serio este problema.

\section{LAS PROPUESTAS DESDE LA ECONOMÍA Y EL DERECHO CONSTITUCIONAL}

El derecho y la economía han caminado paralelamente desde que se fundaron las primeras cátedras de Economía en todas las facultades de derecho en el siglo XIX en Europa (Schumpeter ${ }^{7}, 2004:$ 792-793, 805). Además de la jurisprudencia, la sociología, la historia y la psicología son otras ciencias sociales imprescindibles para el análisis económico (2004: 747). Como diría Schumpeter, la economía es «un gran ómnibus que acarrea numerosos pasajeros de intereses y capacidades inconmensurables» ibid.: 793).

Por otro lado, el modelo económico de un Estado queda definido en su constitución y desde esa perspectiva el derecho constitucional se relaciona con la economía, especialmente en nuestro caso desde esa parte llamada la constitución económica, y desde otros espacios donde se recogen valores y principios como el preámbulo o el título preliminar, así como también en el título I, donde se recogen los derechos. La evolución de la configuración del poder público ha estado siempre vinculada a la evolución de la economía a través del marco fundamental del derecho constitucional (Gordillo, 2019); por tanto, la evolución de modelo económico debe reflejarse también en el orden constitucional.

\section{DESDE LA ECONOMÍA FEMINISTA}

La economía no puede analizarse como un compartimento estanco, sino en relación con otras áreas del conocimiento que le proporcionan el sustento

7 La edición consultada es la de la Fundación ICO, Ariel. La edición original fue publicada en 1954 por Oxford University Press. 
y el contexto para su estudio; pero además, actualmente es preciso analizar la economía desde enfoques diferentes al tradicional basado en el homo economicus. Este concepto, propuesto por Adam Smith en La riqueza de las naciones (1776), de modelo racional y universal de la economía que trata de satisfacer sus deseos y buscar riqueza y utilidad con el menor coste y esfuerzo posible, sería más tarde completado por Stuart Mill $(1836,1844)$ con la búsqueda del bienestar. En definitiva, ese modelo de conducta humana que ha venido definiendo el pensamiento económico desde entonces, se ha demostrado insuficiente porque no puede ser universal ni tiene en cuenta otros aspectos necesarios para la comprensión completa del comportamiento económico.

La economía feminista utiliza la metáfora de la generación espontánea del hombre económico (el «hombre hongo»), como si no hubiera tenido infancia o vejez (Benería et al., 2018: 97) ni necesidades de cuidado de ningún tipo, y destaca que para los economistas estas no entraban en la ecuación. Como dice Katrine Marçal, «de la mano invisible de Adam Smith nació un hombre económico» que tomaba decisiones y las ejecutaba para satisfacer sus deseos de obtener riqueza y utilidad sin tener en cuenta que para ello era imprescindible la intervención de otras personas y el desempeño de otras ocupaciones. «Adam Smith logró solo a medias responder a la pregunta fundamental de la economía, ya que si tenía asegurada la comida no era solo porque los comerciantes sirvieran a sus propios intereses por medio del comercio, sino también porque su madre se encargaba de ponérsela en la mesa todos los días» (Marçal, 2016: 28). La labor desempeñada por las mujeres a lo largo de la Historia, necesaria para el sostenimiento de la vida nunca ha contado para la economía. Paradójicamente, la

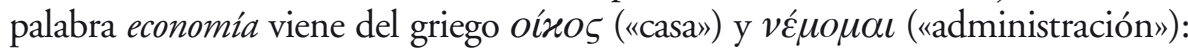
oikonomos («administración del hogar»), y son las mujeres las que se han ocupado de la gestión de los hogares, labor imprescindible para el desarrollo económico.

Según el estudio de María Ángeles Durán (2018: 373), «ninguna otra actividad de la economía española consume tanto trabajo como el cuidado. Sin embargo es invisible en la Contabilidad Nacional». Como he comentado anteriormente, el trabajo de cuidados realizado por las mujeres para sostener la vida — que afecta no solo a cuestiones de salud y de bienestar, sino que además se extiende a todos los aspectos de la convivencia (ibid.: 373) — no cuenta para la economía; por tanto, la contabilidad económica queda incompleta.

Si se quiere tener una visión completa de la economía no se puede ignorar lo que la mitad de la población hace la mitad del tiempo (Marçal, 2016: 67). El trabajo de las mujeres (o los trabajos de cuidados sin considerar quien lo ejerce, aunque sabemos que son mayoritariamente ejercidos por las mujeres, también ahora) se da por descontado, es «una estructura invisible e indeleble» (id.). Como las tareas domésticas son cíclicas, no crean bienes tangibles que se puedan comercializar y, por tanto, no se consideran una tarea económica. 
La economía feminista pone en valor las actividades que no producen bienes tangibles, pero que son imprescindibles para mantener la vida, las familias; en definitiva, la estructura social. La idea de que sin aquellas la vida no se sostiene tiene que ser el fundamento y la justificación de las políticas socioeconómicas.

Existen varias propuestas que afloran para devolver a las tareas de cuidado la importancia que merecen. En este trabajo nos centraremos especialmente en aquellas que se reivindican desde el feminismo como movimiento que tiene como objetivo alcanzar la justicia social en igualdad.

\subsection{Una renta de cuidados}

Desde una perspectiva económica y política, la sociedad descansa sobre dos pilares fundamentales: la producción de bienes que necesitamos para nuestra subsistencia y la producción de "nosotros mismos» porque nos necesitamos (Jónasdóttir, 1993: 53-65). Sin embargo, lo que los hombres controlan y explotan de ese modo de producción por el que nos relacionamos no es el trabajo de las mujeres y el poder del trabajo, sino el amor y el poder de vida resultante de ese modelo de producción (Jónasdóttir, 1993: 70). Los vínculos y dependencias económicas, jurídicas, etc. que han existido entre mujeres y hombres a través de la historia se han debilitado, quedando solo el vínculo natural: «la dependencia del amor», de la que la necesidad de cuidados es una de sus manifestaciones.

Como argumenta Katrine Marçal (2016: 127), la economía debería haberse organizado en torno a lo que es importante para la gente. En lugar de eso lo que se hizo fue «redefinir a las personas para que se ajustaran a una idea de economía», y si «de verdad hubiésemos querido preservar el amor y el cuidado en nuestras sociedades», en lugar de apartarlos de la idea central de economía «deberíamos haberlos apoyado con dinero y recursos».

Las reivindicaciones de una renta que reconozca la centralidad del trabajo de reproducción y de cuidados y retribuya el trabajo feminizado e invisibilizado están desde hace tiempo muy presentes, y más ahora en plena crisis económica y sanitaria derivada de la COVID-19. La mercantilización de la vida no es compatible con las actividades de cuidado y con la preocupación por resituar la vida en el centro de la política. Como explica Giacomo D'Alisa (2020), quizás no haya que elegir, pero sí priorizar y buscar el equilibrio necesario para dar valor al cuidado.

El enfoque por una renta de cuidados no se basa en un derecho abstracto universal a una renta mínima como hace el enfoque de la renta básica universal, sino en la labor de cuidado que se ejerce diariamente. No se demanda una renta sin importar la contribución a la producción del valor social, sino más bien se reivindica la parte de valor social a la cual se contribuye con el 
trabajo de cuidado; un trabajo que generalmente se vuelve invisible para que sea más fácilmente apropiable. Como explica D'Alisa, el objeto de la renta es la contraprestación por hacer posible una vida mejor para el conjunto de la sociedad y para que el sistema sea sostenible. El fin de reivindicar esta renta es «visibilizar la materialidad de los actos de cuidado y reproducción y su inicua distribución entre los géneros». La campaña internacional por una renta del cuidado surge de la Globalwomenstrike.net, que se define como una red internacional de base que hace campaña por el reconocimiento y el pago de todo el trabajo de cuidado, en el hogar y fuera ${ }^{8}$.

Si bien la remuneración de los cuidados, sobre todo cuando son los propios - es decir de los seres queridos o al propio cargo- es un tema complejo porque la mayoría de las veces es percibido como un preferencia individual (Benería et al., 2018: 297), incluso como un «acto de amor» (como explicaba Jönasdóttir, 1993), hace tiempo que la investigación de la economía feminista comenzó el debate por la valoración del trabajo doméstico o de cuidados, no el ejercido por las profesionales o asalariadas contratadas a tal efecto, sino el no remunerado, el llamado "trabajo reproductivo» (Benería et al.: 286), insistiendo en la importancia de documentarlo y contabilizarlo. Es preciso destacar que se empleó este concepto de trabajo reproductivo para resaltar el valor vital de esta forma de trabajo no remunerado para la «reproducción de la fuerza de trabajo, actual y futura». Su visibilidad tiene una repercusión en el incremento de su valor, y por otro lado sirve para analizar las desventajas que genera para quien lo ejerce y compensar los desequilibrios mediante las políticas de conciliación y corresponsabilidad.

\subsection{Un sistema estatal de cuidados}

Las políticas públicas recientes han ido consolidando a través del marco normativo (especialmente sobre conciliación, corresponsabilidad, flexibilización de los tiempos, igualdad de oportunidades) un modo diferente de atender a las necesidades de cuidado de manera más solidaria y corresponsable. El

8 «Las madres y otros cuidadores o cuidadoras tienen derecho a un salario digno [...]. La codicia corporativa y la conquista militar nos están empobreciendo, destruyendo el clima y el mundo. Hacemos campaña por una economía solidaria en la que los seres humanos y todas las vidas son centrales, no un medio para llegar a un fin. Hacemos campaña para poner fin a las relaciones de poder de sexo, raza, clase, ingresos, nación, estatus migratorio, ciudad y campo, edad, discapacidad, ocupación, identidad de género, Norte y Sur [...] (véase las organizaciones que forman parte de GWS). Trabajamos con Payday, una red de hombres que comparten nuestros objetivos». Este es el núcleo principal del manifiesto que puede verse en: https://globalwomenstrike.net/. 
mencionado «Real Decreto Ley 6/2019, de 1 de marzo, de medidas urgentes para garantía de la igualdad de trato y de oportunidades entre mujeres y hombres en el empleo y la ocupación» constituye un avance importante por la igualdad efectiva al permitir, entre otras medidas, la equiparación de los permisos de maternidad y paternidad, pero es solo una de las manifestaciones de lo que podría ser un sistema estatal de cuidados.

La crisis sociosanitaria de la COVID-19 ha puesto en evidencia no solo la vulnerabilidad de las personas, sino también la fragilidad del sistema de cuidados: de los y las menores a cargo de madres trabajadoras que no han podido atender sus obligaciones profesionales en igualdad de condiciones; de las profesionales del cuidado que no han podido ejercerlo por el confinamiento, dejando desatendidas personas necesitadas; del personal médico y de enfermería que se han visto desbordados sin medios, y del sector más frágil que se ha mostrado en la crisis, las residencias y centros de personas mayores. En definitiva, una débil red de cuidados.

La comisión para la reconstrucción social y económica ha llamado a comparecer al grupo de trabajo Cuidados y COVID-199, cuya finalidad es lograr la constitución de un sistema estatal de cuidados partiendo de un plan de arranque con un triple objetivo: el compromiso institucional, generar un debate social y dar respuesta a situaciones de emergencia.

Las medidas de emergencia que se plantean ante la crisis COVID abarcan cuatro grandes áreas: las relativas al empleo en el hogar, los cuidados de hijas e hijos, los cuidados de las personas mayores y dependientes, y las relativas a garantizar el empleo en condiciones dignas, creando empleo público en el ámbito de los cuidados (dependencia, residencias mayores, escuelas infantiles $0-3$, y atención en domicilio).

Y las políticas específicas que deberían desarrollarse para garantizar un derecho universal al cuidado estarían vinculadas a tres ámbitos: la reorganización del sistema de servicios de cuidados, la reformulación y ampliación de las prestaciones y la profesionalización de los cuidados en precario (cuidadoras no profesionales). En definitiva, las políticas faro que deben guiar la orientación del nuevo modelo están básicamente enfocadas a un marco normativo específico: la ley de cuidados y sostenibilidad de la vida y la ley de tiempos.

9 Aportación feminista al debate de la reconstrucción post-COVID-19. Hacia un sistema estatal de cuidados. Documento elaborado por el grupo de trabajo Cuidados y COVID-19, en el que han participado Yayo Herrero, Toni Morillas, Amaia Pérez Orozco, Carmen Castro, Inés Campillo, Bibiana Medialdea, María Eugenia R. Palop, Laura Gómez, Ángela Rodríguez, Cristina Simó, Amanda Meyer, Nora García, Esther López Barceló, Haizea Miguela y Clara Alonso. 
La primera tendría como objeto principal la identificación de los servicios y actividades esenciales para la sostenibilidad de la vida, mientras que la segunda, la fijación de los criterios para disolver la división sexual del trabajo y la regulación del reparto y los usos del tiempo (vitales, laborales, de crianza y cuidados y de ciudadanía). Esta propuesta de marco normativo constituiría la base para la transición a un modelo económico pensado para sostener la vida. El contrapunto para sostener la economía estaría en la responsabilidad individual y colectiva de asumir funciones y responsabilidades con las demás personas con quienes nos relacionamos.

\section{DESDE EL DERECHO CONSTITUCIONAL}

Desde el derecho constitucional se han hecho también propuestas referidas sobre todo al cambio constitucional, que se dirigen, por un lado, a una revisión de los derechos sociales, dotándoles de mayores garantías y ampliando su interpretación por la vía jurisprudencial, y por otro, al reconocimiento de nuevos derechos fundamentales sociales.

Universalizar los derechos fundamentales, no solamente los de libertad, sino también los sociales, es un tema pendiente en el constitucionalismo, pero no solo, también lo es limitar el control ejercido por los poderes económicos imponiendo determinadas condiciones al Estado en detrimento de las garantías de los derechos sociales, afectando así la fuerza normativa de la Constitución (Balaguer, 2018).

\subsection{Los derechos sociales fundamentales}

El preámbulo de la Constitución española proclama «el progreso de la cultura y de la economía para asegurar a todos una digna calidad de vida». Uno de los instrumentos más potentes para lograrlo es el reconocimiento de una serie de derechos que se hallan garantizados en el título I, aunque el problema es que no con la misma intensidad, es decir, no con las mismas garantías.

Según las garantías constitucionales, la Constitución española distingue tres grupos de derechos: el primer nivel estaría integrado por los derechos fundamentales, los que pueden gozar de la protección reforzada del recurso de amparo (arts. 14-30); el segundo se refiere a aquellos considerados derechos subjetivos, pero que quedan fuera de esta protección (arts. 31-38), y el tercero se correspondería con los que quedan ubicados en el capítulo III, los principios rectores de la política social y económica (arts. 39-52). Sin embargo, esta distinción que se hizo en 1978 no responde a la realidad de 2020, especialmente porque algunos derechos que se ubican fuera de la sección primera son tan fundamentales como los incluidos en ella. Son los llamados derechos sociales, que bien 
podrían llamarse derechos sociales fundamentales, y que se encuentran tanto en la sección segunda del capítulo II, como en el capítulo III dentro de los principios rectores de la política social y económica, muchos de los cuales están ya enunciados como derechos (salud, vivienda). En todo caso, es posible defender el carácter fundamental de los derechos sociales partiendo de la indivisibilidad de todos los derechos humanos (Jimena Quesada, 2012: 1408). Según Ferrajoli (2019: 16), entre los derechos fundamentales se distinguen los derechos individuales de libertad y los derechos sociales, que son los que sirven para remover o reducir las desigualdades sociales, de manera que la igualdad jurídica se identifica con el universalismo de los derechos fundamentales en el sentido de que son derechos indivisibles que corresponden igual y universalmente a todas las personas.

Estos derechos se corresponden muy bien con el mencionado enfoque de los derechos de Balakrishnan y Elson (2011), que permite realizar la evaluación de las políticas públicas a partir de los derechos, considerando que existen unos mínimos esenciales en los derechos económicos y sociales que es preciso preservar en todos los procesos económicos (Benería et al., 2018: 72). Por otro lado, pueden vincularse los derechos sociales, muchos de los cuales se hallan incluidos en los principios rectores, con los Objetivos de Desarrollo Sostenible (ODS). «El corazón de los ODS se relaciona con el constitucionalismo social y este en el caso español tiene su más fiel reflejo en los principios rectores de la política social y económica» (Jimena, 2019: 34).

Se ha escrito mucho sobre la revisión de los derechos sociales ${ }^{10}$ y de la necesidad de dotarles de reconocimiento y de las máximas garantías como uno de los retos pendientes para la ciudadanía (Rubio, 2013: 206). Se ha propuesto por parte de la doctrina constitucional dotar a todos los derechos de la consideración de derechos fundamentales ${ }^{11}$, también por parte de los partidos políticos y de organizaciones no gubernamentales (Marrades Puig, 2016: 222). Ello podría hacerse por la vía de la interpretación y, cuando fuera posible, por medio de una reforma constitucional.

En la propuesta de reforma constitucional de Rodríguez Ruiz y Gómez Fernández $(2018: 129,130)$ se constata la necesidad de sacar del capítulo III algunos auténticos derechos, como son el derecho a la salud en un medioambiente sano y sostenible (Rodríguez Ruiz, Marrades Puig y León Alonso 2018: 124, 125) o el

10 A estos efectos, puede verse Derechos sociales y principios rectores. Actas del IX Congreso de la Asociación de Constitucionalistas de España. No es objeto de este trabajo el análisis de los derechos sociales pero sí en relación a las posibilidades para dotarles de las máximas garantías.

11 Red Feminista de Derecho Constitucional. Posicionamiento de la RFDC ante la necesaria reforma de la Constitución. (4/12/2017) (disponible en: https://bit.ly/389Ftnw). 
derecho a la vivienda (ibid:: 124-125) para formar parte del capítulo II junto con otros derechos de nueva creación.

Es preciso hablar de la inclusión de nuevos derechos que llenen el vacío que las nuevas exigencias sociales demandan. Hace algún tiempo que vienen reivindicándose, entre ellos el derecho al cuidado (Marrades Puig, 2016; 2018: 105; 2019: 21 y ss.), y ahora ante la emergencia y la crisis provocada por la COVID-19 no hay duda de que hay que afrontar este reto. El grupo de trabajo de Cuidados y COVID-19 en su comparecencia ante la Comisión para la Reconstrucción Social y Económica presentó los presupuestos para una ley estatal de cuidados que debería ir vinculada a un derecho al cuidado. El fin de la ley y del reconocimiento del derecho al cuidado es obtener el compromiso de los poderes públicos para priorizar el cuidado y la atención a las personas en igualdad de condiciones por encima de otros intereses económicos. Es un proyecto que permite la transición de modelo económico mediante un proceso que puede ser más o menos radical, pero necesario para erradicar desigualdades. Como señala Ferrajoli en su prólogo al Manifiesto por la igualdad (2019), el proyecto de la igualdad —que conlleva la promoción del interés de todos-, puede convertirse en el nuevo sustrato de reconstrucción de la política: desde arriba como programa reformador y como proyección de las promesas constitucionales, mediante la introducción de límites no solo a los poderes públicos, sino también a los poderes privados del mercado, en garantía tanto de los derechos individuales de libertad como de los derechos sociales; y desde abajo como motor de la participación y movilización política al ser la igualdad en los derechos fundamentales un factor de recomposición solidaria de la disgregación social producida por el dominio de los mercados.

\subsection{La jurisprudencia en igualdad}

Mediante su jurisprudencia, el Tribunal Constitucional ha ido completando el concepto de igualdad, conjugando la igualdad formal del art. 14 con la promoción de la igualdad real o efectiva del 9.2., especialmente en relación con la igualdad de género, y como afirma M. Luisa Balaguer (2019: 75), «ha contribuido a cambiar de manera muy importante la jurisprudencia conceptual en que se venía desenvolviendo la jurisdicción ordinaria, fundamentalmente en los primeros años de la justicia constitucional». Sin embargo, como acertadamente señala Itziar Gómez (2019: 79), «la jurisprudencia constitucional no distingue las desigualdades basadas en el sexo de cualesquiera otras discriminaciones fundadas en causas sociales, ideológicas, personales y de cualquier otro tipo», ni tampoco «realiza una construcción específica sobre el género, sino que habla indistintamente de sexo y género (STC 159/2016), a pesar de que parece apreciarse cierta conciencia de la distinción en la STC 59/2008». 
De acuerdo con Itziar Gómez, creo que no se puede juzgar la distinción por razón de género con el mismo método racional con que se juzga las demás, «buscando la causa de la discriminación y las razones que justifican su existencia, para valorar después si tal justificación es constitucionalmente admisible o no lo es», ya que ese método analiza el caso perdiendo de vista el contexto de discriminación estructural en que se desarrolla la vida de las mujeres, por lo que no puede producir los mismos resultados. Esta reflexión describe una parte reciente de la jurisprudencia constitucional en materia de igualdad social que ha supuesto una involución a la hora de interpretar el principio de igualdad debido a este error de enfoque. No obstante, la producción de algunos votos particulares ha contribuido a matizar estos errores de interpretación de la igualdad social con perspectiva de género, especialmente en materia de cuidados.

Uno en concreto, el emitido al fallo de la STC 117/2018, de 29 de octubre de 2018 (Recurso de Amparo 6299-2017), pone en valor la necesidad de interpretar la igualdad en relación con la corresponsabilidad dentro de la familia y en el marco de una sociedad que está en transformación. «La interpretación que formula el Tribunal, y que no deja de traer al centro del análisis el reparto equitativo de las responsabzilidades familiares, olvida que no se trata solo de la corresponsabilidad en el ámbito familiar, sino de la repercusión externa que la asunción de responsabilidades familiares tiene en el ámbito laboral» porque obviamente tiene una repercusión social al desincentivar la contratación de mujeres en un periodo de edad determinado.

Merece la pena transcribir literalmente el párrafo donde la magistrada Balaguer argumenta que "la Sentencia ignora que existe un efecto claro de discriminación indirecta de las mujeres, asociado al hecho de la maternidad, que el legislador debiera tratar de erradicar por mandato del artículo 9.2 CE. Un Tribunal Constitucional de este siglo debería haber reconocido la necesaria evolución de la realidad social, y profundizado en el análisis de los efectos reales de las medidas de protección que aquí se cuestionan».

Balaguer se refiere a la STC 128/1987 como el hito histórico que supuso en la jurisprudencia constitucional sobre igualdad, pero tres décadas después $\mathrm{y}$ tras cambios sustanciales,

sigue existiendo una fractura clara entre hombres y mujeres en el mercado laboral... Hoy ya no se trata solo de lamentar que la mujer concentre la mayor parte de cargas derivadas del cuidado de la familia, y particularmente del cuidado de los hijos, porque sigue haciéndolo a pesar de los avances innegables. Se trata de analizar por qué las medidas desarrolladas para compensar esa realidad social, destinadas fundamentalmente a las mujeres, no logran superar como debieran esa realidad y no aseguran la igualdad real de las mujeres en el acceso al mundo laboral y su promoción dentro del mismo. Se trata de examinar por qué esas medidas no logran atajar el problema del desigual reparto de los desincentivos entre los hombres y las mujeres. 
Por eso, dice Octavio Salazar (2019: 88) que esta sentencia es un ejemplo de cómo el TC no considera la dimensión relacional que el género conlleva ni las estructuras sociales y económicas que siguen dividiendo la ciudadanía en la esfera pública masculina y la privada femenina. Salazar analiza de forma crítica que la sentencia no aluda a los criterios interpretativos de la LO 3/2007 ni incorpore en sus razonamientos las herramientas que el feminismo jurídico lleva décadas aportando para hacer eficaz, también desde el punto de vista de la interpretación y la aplicación del derecho, el mandato del art. 9.2 CE. A ello se suma también García Campá (2019: 14) al señalar la evidente tensión que en ocasiones se desprende entre la LO 1/2007, de 22 de marzo, para la Igualdad Efectiva de Mujeres y Hombres y la propia Constitución, entre el concepto de igualdad de la ley (art. 1.11) y los límites a la discriminación por razón de sexo del artículo $14 \mathrm{CE}$.

Creo, siguiendo la reflexión de Juana Gil Ruíz (2019: 73), que uno de los grandes retos que se nos plantea en el ámbito laboral consiste en «encontrar un modelo satisfactorio para mujeres y hombres coherente con las expectativas de la vida familiar y profesional, más aún ante las profundas transformaciones que están afectando al modo en que se relacionan estos en dichos ámbitos». Justamente la clave está en el modo de relacionarnos y en las expectativas que tenemos, que no son más que las dignas aspiraciones de tener una vida plena en igualdad de condiciones en el ámbito personal, familiar y laboral-profesional, pero también de participar en la vida pública más allá del trabajo, eso es, en el ámbito político, social y cultural. Es necesario lograr esta comprensión, un anhelo de tantos siglos para las mujeres ahora también compartido por muchos hombres que sean capaces de abordar con determinación este reto de la igualdad en sus relaciones personales y laborales y necesariamente desde sus puestos en las instituciones públicas. Es uno de los elementos indispensables para esta nueva era de la igualdad que parece se está fraguando. La crisis sociosanitaria de la COVID-19 ha supuesto un punto de inflexión para actuar sobre los problemas que ya existían y que ahora se han manifestado de manera todavía más intensa.

\section{A MODO DE EPÍLOGO: ¿̇UNA NUEVA ERA?}

Se escuchan voces que afirman que estamos ante el comienzo de una nueva era o que claman por ella. Es pronto para hacer afirmaciones rotundas, pero lo cierto es que estamos atravesando grandes transformaciones.

Según el Diccionario RAE, era significa: «Período de tiempo que se cuenta a partir de un hecho destacado» y también: «Extenso período histórico caracterizado por una gran innovación en las formas de vida y de cultura». 
Acogiéndonos al primer significado de la palabra, y en el caso que nos ocupa es obvio que estamos comenzando un nuevo período de un tiempo convulso e incierto de crisis social, económica y seguramente cultural a partir de un hecho muy destacado como la epidemia o pandemia, si lo explicamos desde una perspectiva global, causada por la COVID-19, con un dramático impacto en la vida de las personas. Si nos referimos al segundo significado, nos faltaría el elemento tiempo ("extenso período histórico») para poder observar a largo plazo si la crisis sanitaria y económica producida por la COVID-19 ha provocado una "gran innovación en las formas de vida y cultura». Aun así, esta «innovación» es ya perfectamente palpable y visible.

Es evidente que somos ahora más conscientes que nunca de nuestra vulnerabilidad y por tanto más conscientes de la necesidad de seguridad, a la vez que tenemos muy presente la importancia de garantizar la libertad. Es un equilibrio que debe obtenerse conjugando los valores de solidaridad y de cuidado con los ya reconocidos por nuestro Estado social y democrático de derecho, de igualdad y libertad, recuperando la proclama de nuestro preámbulo constitucional: «Promover el progreso de la cultura y de la economía para asegurar a todas las personas una digna calidad de vida». Pero el progreso de la economía no puede darse mientras sigan rigiendo las reglas tradicionales del mercado y el productivismo, desconsiderando que otros valores cuentan para la economía, que son en definitiva los esenciales para una vida digna. Necesitamos un cambio cultural que recupere el valor de la persona y que reoriente la escala de valores. Este tiempo de confinamiento nos ha hecho más conscientes de las cosas importantes y de las cosas prescindibles, nos ha hecho más conscientes de lo que debe contar, de la jerarquía de los valores, incluso de la jerarquía de las profesiones y ocupaciones, de lo que debe ocupar un papel central en la economía y en la política, incluso de la importancia de vivir en un planeta más limpio, y todo ello sí que nos aproxima al cambio cultural. Es esperable que la fuerza del poder económico trate de impedirlo, pero es preciso apuntalar las estructuras del cambio y no retroceder; hemos arriesgado demasiado. Como manifestaba Ferrajoli en el primer período del confinamiento, "esperemos que esta emergencia del coronavirus provoque un despertar de la razón, generando la plena consciencia de nuestra fragilidad y de nuestra interdependencia global [...] y de la falta de adecuadas instituciones globales de garantía» (García Jaén, 2020).

Ahora que aquel está tocando a su fin, estamos ya en condiciones de determinar los fallos del sistema. Si se quiere preservar el cuidado a las personas, resulta imprescindible dotar de medios económicos a las instituciones de cuidado para que un rebrote de la pandemia o cualquier otra situación similar no sature el sistema sanitario. Pero a la vez hay que reforzar el sistema educativo para equilibrar el igual acceso al mismo de todas las personas ante una situación como la vivida, y no solo desde una perspectiva económica, 
sino también como vehículo para transmitir los valores de ese cambio; entre estos también debe estar el sentido de responsabilidad que todas las personas debemos asumir para cuidar y cuidarnos.

En el momento de cierre de este trabajo, recién finalizado el extenso periodo de estado de alarma ${ }^{12}$, la pandemia COVID-19 sigue amenazándonos con rebrotes de la enfermedad, lo que hace necesario seguir apelando al sentido de la responsabilidad individual y colectiva. Parece ser que tendremos que acostumbrarnos a vivir entre la inseguridad y la incerteza. Por ello deviene imprescindible contar con un sistema sociosanitario fuerte y apoyado por un diseño económico de políticas públicas que priorice la atención a las personas.

\section{Bibliografía}

Balaguer Callejón, F. (2018). Las dos grandes crisis del constitucionalismo frente a la globalización en el siglo xxi. Revista de Derecho Constitucional Europeo, 30, 2.

Balaguer Callejón, M. L. (2019). Encuesta sobre igualdad entre hombres y mujeres. Teoría y Realidad Constitucional, 43, 15-99. Disponible en: https://doi. org/10.5944/trc.43.2019.24398.

Balakrishnan, R. y Elson, D. (2011). Introduction: Economic Policies and Human Rights Obligations. En R. Balakrishnan y D. Elson (eds.). Economic Policies and Human Rights (pp. 1-27). Londres: Zed Books.

Bathia, A. (2020). Las mujeres y el covid 19: cinco acciones que los gobiernos pueden adoptar sin demora. ONU Mujeres. Disponible en: https://bit.ly/2HXUf5B.

Benería, L., Berik, G. y Floro, M. (2018). Género, desarrollo y globalización. Una visión desde la economía feminista. Barcelona: Bellaterra

Benlloch, C. y Aguado, E. (2020). Teletrabajo y conciliación. El estrés se ceba con las mujeres. The Conversation [blog] 29-4-2020. Disponible en: https://bit. ly/389wJ0K.

Camps, V. (2000.) El siglo de las mujeres. Madrid: Cátedra

Cascajo Castro, J. L., Terol Becerra, M. J., Domínguez Vila, A. M. y Navarro Marchante, V. J. (coords.) (2012). Derechos sociales y principios rectores. Actas del IX Congreso de la Asociación de Constitucionalistas de España. Valencia: Tirant lo Blanch.

Durán Heras, M. Á. (2018). La riqueza invisible del cuidado. Valencia: Universitat de València.

Ferrajoli, L. (2019). Manifiesto por la igualdad. Madrid: Trotta.

García Campá, S. (2019). Criar sin parir. Sexo y género como categorías de análisis jurídico en la Sentencia del Tribunal Constitucional 111/2018, de 17 de octubre. Revista de Trabajo y Seguridad Social, 433, 1-22

12 El 20 de junio de 2020 el presidente del Gobierno, Pedro Sánchez, comparecía en Moncloa para declarar el fin del estado de alarma el 21 de junio de 2020 (disponible en: https://bit.ly/34THvGk). 
Gil Ruiz, J. M. (2019). Repensando la Constitución: una mirada al deber de trabajar y al derecho al trabajo. Revista de Derecho Politico, 104, 59-85. Disponible en: https://doi.org/10.5944/rdp.104.2019.24309.

Gilligan, C. (1982). In a Different Voice. Psychological Theory and Women's Development. Cambridge: Mass, Harvard University Press.

Gilligan, C. (1985). La moral y la teoría, psicología del desarrollo femenino. México: Fondo de Cultura Económica.

Gilligan, C. (1993). Replay to critics. En M. J. Larrabee (ed). An ethic of Care. Londres: Routledge.

Gómez Fernández, I. (2019). Encuesta sobre igualdad entre hombres y mujeres. Teoría y Realidad Constitucional, 43, 15-99. Disponible en: https://doi.org/10.5944/ trc.43.2019.24398.

Gordillo Pérez, L. y Rodríguez Ortiz F. (2019). Constitución económica y gobernanza económica de la Unión Europea. Cizur Menor: Thomson Reuters-Aranzadi.

Jimena Quesada, L. (2012). La tutela de los derechos sociales: el espacio de la Unión y del Consejo de Europa. En J. L. Cascajo Castro, M. Terol Becerra, A. Domínguez Vila y V. Navarro Marchante (coords.). Derechos Sociales y principios rectores, Actas del IX Congreso de la Asociación de Constitucionalistas de España. Valencia, Tirant lo Blanch.

Jimena Quesada, L. (2019). El constitucionalismo social y los objetivos de desarrollo sostenible, Lex Social, 9, 1, 13-45.

Jónasdóttir, A. (1993). El poder del amor, ¿le importa el sexo a la democracia?. Madrid: Cátedra.

León Alonso, M. (2018). Artículo 15 y Artículo 34. En Y. Gómez Sánchez (coord.) Estudios sobre la reforma de la constitución de 1978 en su cuarenta aniversario (pp. 88-95 y 124-126). Cizur Menor: Aranzadi Thomson-Reuters.

López Guerra, (1983). El modelo económico en la Constitución. Revista de Estudios Económicos y Empresariales, 2, 17-25.

Marçal, K. (2016). ¿Quién le hacía la cena a Adam Smith? Barcelona: Penguin.

Marrades Puig, A. (2016). Los nuevos derechos sociales: el derecho al cuidado como fundamento del pacto constitucional. Revista de Derecho Político, 97, 209-242. Disponible en: https://doi.org/10.5944/rdp.97.2016.17623.

Marrades Puig (2018). Artículo 26 y Artículo 34. En Y. Gómez Sánchez (coord.) Estudios sobre la reforma de la constitución de 1978 en su cuarenta aniversario (pp. 105-107 y 124-126). Cizur Menor: Aranzadi Thomson-Reuters.

Marrades Puig, A. (2019). La ética del cuidado, la igualdad y la diversidad: valores para una constitución del siglo xxi. En A. Marrades Puig (coord.). Retos del estado constitucional del siglo XXI: derechos, ética y políticas del cuidado (pp.17-39). Valencia: Tirant lo Blanch.

Mill, J. S. (1836). On the Definition of Political Economy, and on the Method of Investigation Proper to It. London and Westminster Review, 4, 120-164.

Mill, J. S. (1844). Essays on Some Unsettled Questions of Political Economy. London: Longmans, Green, Reader and Dyer.

Moltó Carbonell, M. L. y Uriel, E. (2008). El trabajo doméstico cuenta: las cuentas de los hogares en España. Madrid: Fundación de las Cajas de Ahorros. 
Nussbaum, M. (2003). Capabilities as Fundamental Entitlements: Sen and Social Justice. Feminist Economics 9 (2-3), 33-59. Disponible en: https://doi.org/10.1 080/1354570022000077926.

Nussbaum, M. (2004). Promoting Women's Capabilities. En L. Beneria, S. Bisnath (ed.). Global Tensions (pp. 241-256). Nueva York: Routledge.

Puleo, A. (2019). Claves ecofeministas para rebeldes que aman la tierra y a los animales. Madrid: Plaza y Valdés.

Rodríguez Ruiz y Gómez Fernández (2018). Los principios rectores de la política social y económica. Nuevos principios rectores de la política social y económica. En Y. Gómez Sánchez (coord.) Estudios sobre la reforma de la constitución de 1978 en su cuarenta aniversario (pp. 129-130) Pamplona: Aranzadi, Thomson-Reuters.

Rubio Castro, A. (2013). Las Innovaciones en la medición de la desigualdad. Madrid: Dykinson.

Salazar Benítez, O. (2019). Encuesta sobre igualdad entre hombres y mujeres. Teoría y Realidad Constitucional, 43, 15-99. Disponible en: https://doi.org/10.5944/ trc.43.2019.24398.

Schumpeter, J. (2004). Historia del análisis económico. Barcelona: Ariel.

Sen, A. (1999) Development as Freedom. New York: Knopf.

\section{Artículos de prensa}

Bassets, M. (2020). Alain Touraine, sociólogo: «Esta crisis va a empujar hacia arriba a los cuidadores», El Pais, 29-3-2020. Disponible en: https://bit.ly/38dYyFb.

D’Alisa, G. (2020). Vital es el "cuidado", no una renta mínima. El Diario.es, 2-62020. Disponible en: https://bit.ly/35VhHsA.

García Jaén, B. (2020). Luigi Ferrajoli, filósofo: «Los países de la UE van cada uno por su lado defendiendo una soberanía insensata». El País, 28-3-2020. Disponible en: https://bit.ly/2TO7Izs.

Kohan, M. (2020). ¿Están respondiendo mejor a la crisis del coronavirus los países gobernados por mujeres? Público, 16-4-2020. Disponible en: https://bit. ly/388ANOw.

Ramonet, I. (2020). La pandemia y el sistema-mundo. Le Monde Diplomatique, 254- 2020. Disponible en: https://bit.ly/3jV4iFG.

Vallejo, I. (2020). Épica del cuidado. El Pais, 15-3-2020. Disponible en: https://bit. ly/3281Msr.

\section{Webgrafía}

https://www.lamoncloa.gob.es/covid-19/Paginas/estado-de-alarma.aspx

https://www.unwomen.org/es/news/stories/2020/3/news-women-and-covid-19governments-actions-by-ded-bhatia

https://globalwomenstrike.net/

http://feministasconstitucional.org/wp-content/uploads/2017/12/RFDC_posicionamiento_RefConst-4-diciembre-2017..pdf

https://www.youtube.com/watch?v=RkOG2JCboTY 